\title{
The galaxy-dark matter connection
}

\author{
Frank C. van den Bosch* \\ Department of Physics, ETH Zürich, Switzerland \\ E-mail: vdboschephys.ethz.ch
}

\section{Xiaohu Yang and H.J. Mo}

Department of Astronomy, University of Massachusetts, USA

\begin{abstract}
What galaxy lives in what halo? The answer to this simple question holds important information regarding galaxy formation and evolution. We describe a new statistical technique to link galaxies to their dark matter haloes, or light to mass, using the clustering properties of galaxies as function of their luminosity. The galaxy-dark matter connection thus established, and parameterized through the conditional luminosity function, indicates the presence of two characteristic scales in galaxy formation: one at $\sim 10^{11} h^{-1} \mathrm{M}_{\odot}$, where galaxy formation is most efficient, and another at $\sim 10^{13} h^{-1} \mathrm{M}_{\odot}$, where a transition occurs from systems dominated by one brightest, central galaxy to systems with several dominant galaxies of comparable luminosity. We test the relation between light and mass established from galaxy clustering alone with dynamical masses obtained from satellite kinematics, and show that both are in excellent agreement. We also present a new (halo-based) galaxy-group finder, and show that the multiplicity function of galaxy groups identified in the 2dFGRS suggests a relatively high mass-to-light ratio on the scales of galaxy clusters, or, alternatively, a relatively low value of the power-spectrum normalization $\sigma_{8}$. These findings are also supported by our studies of pairwise peculiar velocities and satellite abundances. Finally, we directly measure the halo occupation statistics from our galaxy groups, which are a good proxy of dark matter haloes, and show that these are in excellent agreement with our conditional luminosity function model.
\end{abstract}

Baryons in Dark Matter Halos

5-9 October 2004

Novigrad, Croatia

${ }^{*}$ Speaker. 


\section{Introduction}

According to the current paradigm of structure formation, galaxies form and reside inside extended cold dark matter (CDM) haloes. One of the ultimate challenges in astrophysics, therefore, is to obtain a detailed understanding of how galaxies with different physical properties occupy dark matter haloes of different masses. This link between galaxies and dark matter haloes is an imprint of various complicated physical processes related to galaxy formation, such as cooling, star formation, merging, tidal interactions, and a variety of feedback processes. Although the statistical link itself does not give a physical explanation of how galaxies form and evolve, it provides important constraints on these processes and on how their efficiencies scale with halo mass.

To quantify the relationship between haloes and galaxies in a statistical way, it has become customary to specify the so-called halo occupation distribution, $P(N \mid M)$, which gives the probability to find $N$ galaxies (with some specified properties) in a halo of mass $M$. This occupation distribution can be constrained using data on the clustering properties of galaxies, as it completely specifies the galaxy bias, and has been used extensively to study galaxy occupation statistics and large scale structure (e.g., $[2,9,18]$ and references therein).

\section{The Conditional Luminosity Function}

Arguably, one of the most important physical properties of a galaxy is its total luminosity. Ideally, one would therefore consider occupation statistics as a function of luminosity. In particular, this would allow a direct estimate of the average mass-to-light ratios as function of halo mass, as well as the construction of mock redshift surveys. In a series of papers $[12,18]$, we therefore included luminosities in the halo occupation statistics by introducing the conditional luminosity function (CLF) $\Phi(L \mid M) \mathrm{d} L$, which gives the average number of galaxies with luminosities in the range $L \pm \mathrm{d} L / 2$ that reside in a halo of mass $M$. The CLF is the direct link between the galaxy luminosity function $\Phi(L)$ and the halo mass function $n(M)$, according to

$$
\Phi(L)=\int_{0}^{\infty} \Phi(L \mid M) n(M) \mathrm{d} M .
$$

In CDM cosmologies, more massive haloes are more strongly clustered (e.g., [6]). This means that information regarding the clustering strength of galaxies (of a given luminosity) contains information about the characteristic mass of the halo in which they reside. At sufficiently large separations, $r$, the two-point correlation function of galaxies of luminosity $L$ is given by $\xi_{\mathrm{gg}}(r, L)=\bar{b}^{2}(L) \xi_{\mathrm{dm}}(r)$. Here $\xi_{\mathrm{dm}}(r)$ is the dark matter mass correlation function, and $\bar{b}(L)$ is the average bias of galaxies of luminosity $L$, which derives from the CLF according to

$$
\bar{b}(L)=\frac{1}{\Phi(L)} \int_{0}^{\infty} \Phi(L \mid M) b(M) n(M) \mathrm{d} M .
$$

with $b(M)$ the bias of dark matter haloes of mass $M$. Therefore, the combination of an observed luminosity function, $\Phi(L)$, plus measurements of the galaxy-galaxy two-point correlation function as function of luminosity puts stringent constraints on $\Phi(L \mid M)$.

We assume that the CLF can be described by a Schechter function, and describe the mass dependencies using a total of 8 free parameters (see $[12,15,18]$ ). We use a Monte-Carlo Markov Chain 

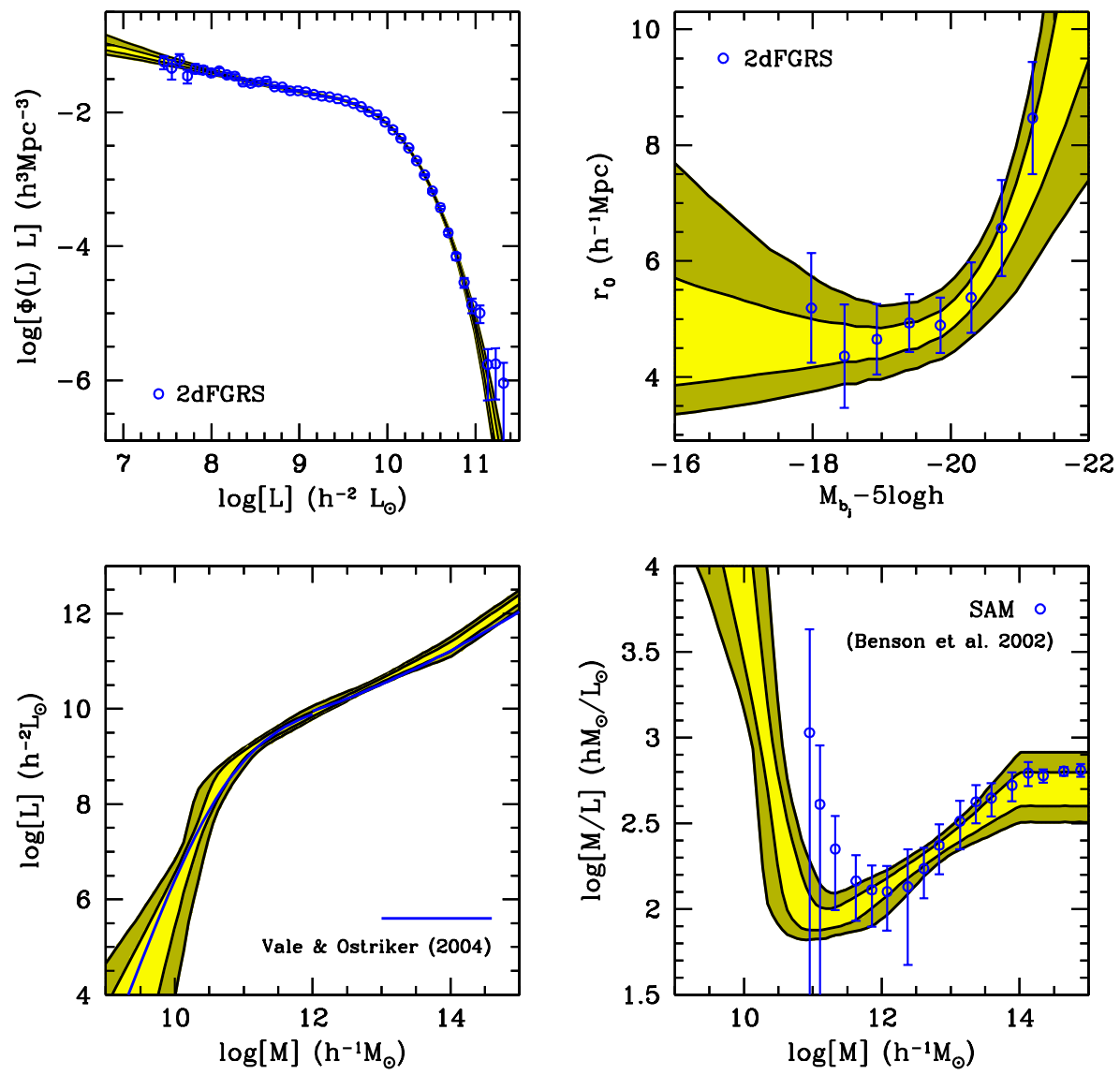

Figure 1: Posterior constraints on a number of quantities computed from the MCMC described in the text. The contours show the $68 \%$ and $99 \%$ confidence limits from the marginalized distribution. Upper left-hand panel: The galaxy luminosity function; open circles with errorbars correspond to the 2dFGRS data from [5]. Upper right-hand panel: galaxy-galaxy correlation lengths as function of absolute magnitude; open circles with errorbars correspond to the 2dFGRS data from [8]. Lower left-hand panel: the total luminosity per halo as function of halo mass. Solid line corresponds to the model of [11], and is shown for comparison. Lower right-hand panel: the average mass-to-light ratio as function of halo mass. Open circles with errorbars correspond to the semi-analytical model of [1] and is shown for comparison. See text for details.

(hereafter MCMC) to probe the likelihood function in our multi-dimensional parameter space, and to put confidence levels on all derived quantities. The results obtained for a $\Lambda \mathrm{CDM}$ 'concordance' cosmology $\left(\Omega_{m}=0.3, \Omega_{\Lambda}=0.7, h=0.7, \sigma_{8}=0.9\right)$ are shown in Fig. 1 . The open circles with errorbars in the upper panels are the data used to constrain the models. The shaded areas indicate the 68 and 99 percent confidence levels on $\Phi(L)$ and $r_{0}(L)$ computed from the MCMC. Note the good agreement with the data, indicating that the CLF can accurately match the observed abundances and clustering properties of galaxies in the 2dFGRS. We emphasize that this is not a trivial result, as the data can only be fitted for a certain combination of cosmological parameters (see [13]).

The lower left-hand panel of Fig. 1 plots the relation between halo mass $M$ and the total halo luminosity $L$, which follows from the CLF according to

$$
\langle L\rangle(M)=\int_{0}^{\infty} \Phi(L \mid M) L \mathrm{~d} L
$$


Note that the confidence levels are extremely tight, especially for the more massive haloes. The $L(M)$ relation reveals a dramatic break at around $M \simeq 10^{11} h^{-1} \mathrm{M}_{\odot}$, indicating a characteristic scale in galaxy formation. These results are in excellent agreement with the $L(M)$ relation of Vale \& Ostriker ([11]), obtained assuming a monotonic relation between light and mass. This agreement combined with the extremely tight confidence levels obtained from our CLF analysis suggests that we have established a robust connection between light and mass.

Finally, the lower right-hand panel of Fig. 1 plots the corresponding mass-to-light ratios as function of halo mass. The pronounced minimum in $\langle M / L\rangle_{M}$ indicates that galaxy formation is most efficient in haloes with masses in the range $5 \times 10^{10} h^{-1} \mathrm{M}_{\odot} \lesssim M \lesssim 10^{12} h^{-1} \mathrm{M}_{\odot}$. For less massive haloes, $\langle M / L\rangle_{M}$ increases drastically with decreasing halo mass, which is required in order to bring the steep slope of the halo mass function at low $M$ in agreement with the relatively shallow faint-end slope of the observed LF. It indicates that galaxy formation needs to become extremely inefficient in haloes with $M \lesssim 5 \times 10^{10} h^{-1} \mathrm{M}_{\odot}$ in order to prevent an overabundance of faint galaxies. The increase in $\langle M / L\rangle_{M}$ from $M \sim 10^{11} h^{-1} \mathrm{M}_{\odot}$ to $M \sim 10^{14} h^{-1} \mathrm{M}_{\odot}$ is thought to be associated with the decreasing ability of the gas to cool with increasing halo mass. The open circles with errorbars correspond to the semi-analytical model of Benson et al. ([1]), which has been tuned to match the galaxy luminosity function. It is extremely reassuring that two completely independent methods yield average mass-to-light ratios that are in such good agreement.

Because it gives a statistical description of the galaxy-dark matter connection, the CLF is an extremely powerful tool. In a series of papers we have used it to investigate large scale structure [16,19], the environment dependence of the galaxy luminosity function [7], the kinematics and abundances of satellite galaxies [14,15], and various properties of galaxy groups [20,21,22]. In addition, we have used the CLF formalism to constrain cosmological parameters [13] and to construct detailed mock redshift surveys [19]. Here we summary a few of the highlights.

\section{Mock Galaxy Redshift Surveys}

An important application of the CLF is the construction of mock galaxy redshift surveys (hereafter MGRSs), which are extremely useful tools to aid in the interpretation of large redshift surveys. As with any data-set, several observational biases hamper a straightforward interpretation of such surveys (e.g., Malmquist bias, redshift-space distortions, fiber collisions). The CLF is ideally suited to build up "virtual" Universes from which mock galaxy redshift surveys can be constructed using the same biases and incompleteness effects as in the real data. In [19] we have used the CLF to populate dark matter haloes in numerical simulations with galaxies of different luminosities, and constructed detailed MGRSs that can be compared on a one-to-one basis with the 2dFGRS (see also $[14,15,20])$.

\section{Satellite Kinematics}

In order to test the relation between light and mass shown in the lower left panel of Fig. 1, and which has been derived solely from the galaxy clustering properties, we use the kinematics of satellite galaxies. Since satellites probe the potential well out to the outer edges of their haloes, they are ideally suited to measure total halo masses (unlike, for example, a disk rotation curve 

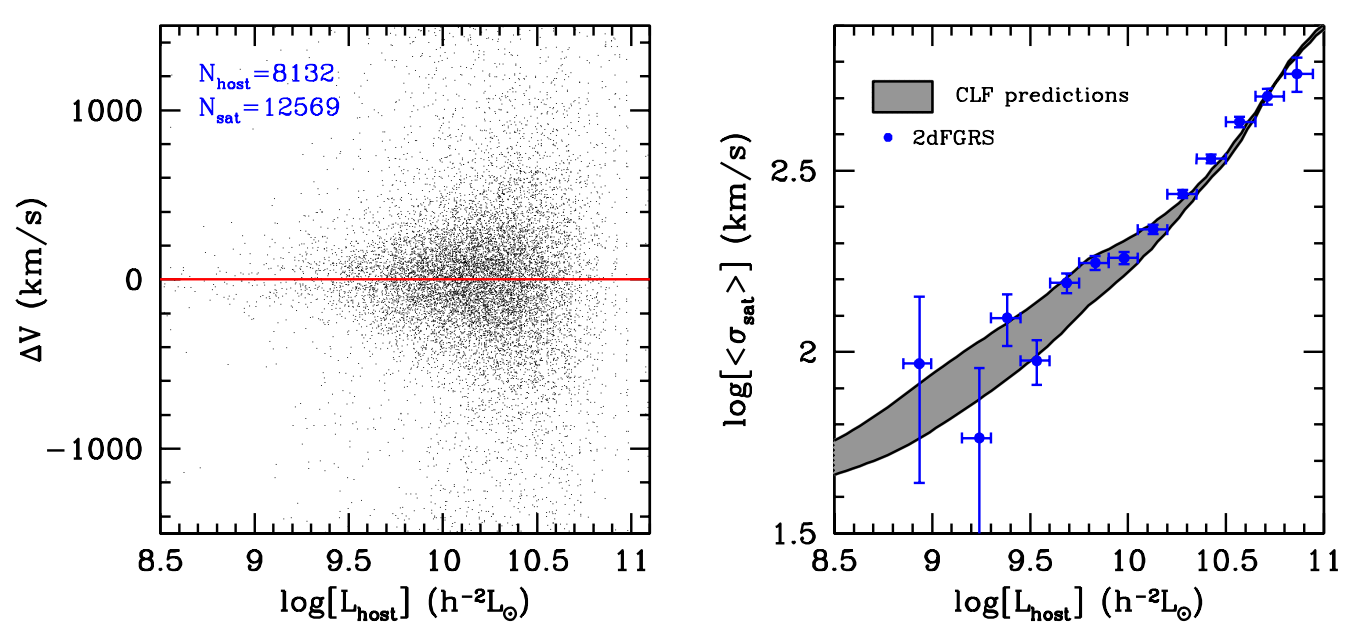

Figure 2: Left-hand panel The difference in line-of-sight velocity, $\Delta V$, between hosts and satellites in the $2 \mathrm{dFGRS}$ as function of the luminosity $L_{\text {host }}$ of the host galaxy. Right-hand panel: Solid dots with errorbars indicate the best-fit $\sigma_{\text {sat }}\left(L_{\text {host }}\right)$ obtained from the 2 dFGRS data shown in the left-hand panel. The gray area indicates the expectation values obtained using the CLF. The excellent agreement with the $2 \mathrm{dFGRS}$ results gives an independent, dynamical confirmation of the relation between light and mass inferred from the galaxy clustering properties.

which typically only probes the potential out to a fraction of the virial radius). A downside of using satellite galaxies, however, is that the number of detectable satellites in individual systems is generally small. One therefore typically stacks the data on many host-satellite pairs to obtain statistical estimates of halo masses (see [3] for an up-to-date review).

Previous attempts to measure the kinematics of satellite galaxies have mainly focussed on isolated spiral galaxies. Using the mock galaxy redshift surveys described above we have investigated to what extent a similar analysis can be extended to include a much wider variety of systems, from isolated galaxies to massive groups and clusters. In particular, we used our MGRSs to optimize the host-satellite selection criteria to yield large numbers of hosts and satellites, and small fractions of interlopers (satellites not physically associated with the halo of the host galaxy). We found that an iterative technique with adaptive selection criteria works best, allowing for an accurate measurement of $\sigma_{\text {sat }}\left(L_{\text {host }}\right)$; see [14] for details.

Applying our adaptive selection criteria to the 2dFGRS yields a total of 8132 host galaxies and 12569 satellite galaxies. The left-hand panel of Fig. 2 plots the velocity difference, $\Delta V$, between host and satellite galaxies as function of host luminosity. Notice the increase of the mean $|\Delta V|$ with increasing $L_{\text {host }}$. Fitting the distributions $P(\Delta V)$ of various luminosity bins with a Gaussian plus constant (to reproduce the true satellites and interlopers, respectively), yields the relation between $\sigma_{\text {sat }}$ and $L_{\text {host }}$ shown in the right-hand panel (solid dots with errorbars). The gray area indicates the expectation values obtained from our CLF where the upper and lower boundaries outline the range of uncertainties due to the unknown second moment of the CLF (see [14] for details). Clearly, the satellite kinematics obtained from the 2dFGRS are in excellent agreement with these predictions. This provides a dynamical confirmation of the average relation between mass and light obtained from our purely statistical CLF formalism! 

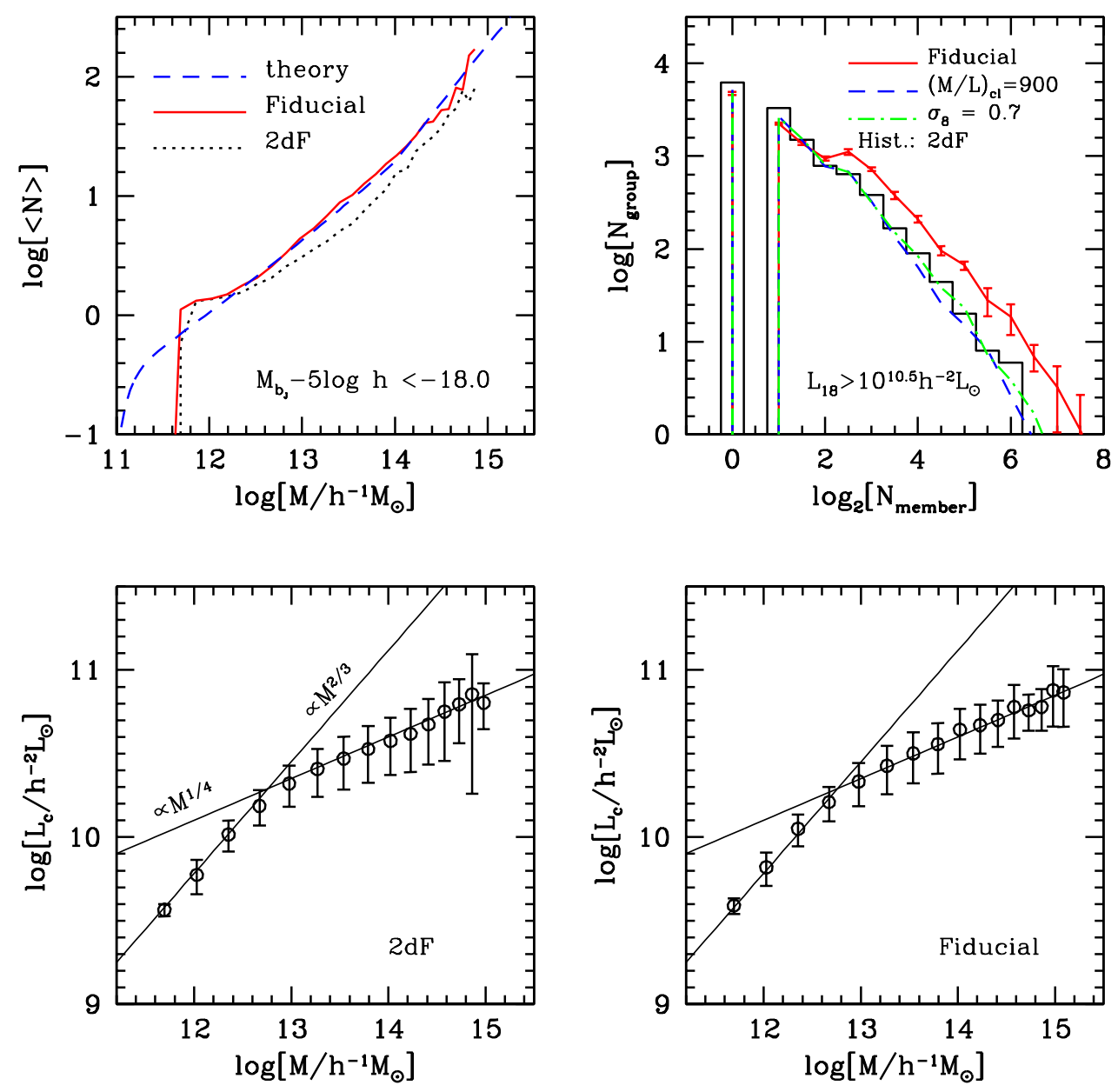

Figure 3: Upper left panel: Mean group occupation numbers as function of halo mass. Dashed line indicates the 'input', as specified by the CLF used to construct the MGRS. Solid and dotted lines indicate results obtained from the groups extracted with our halo-based group finder from the MGRS and the 2dFGRS, respectively. Upper right panel: Number of groups found as function of number of group members. Solid histogram indicates results obtained from the 2dFGRS group catalogue, which is significantly inconsistent with those obtained from our fiducial MGRS. To remedy this discrepancy, either clusters have to have a relatively high mass-to-light ratio of $\sim 900 h(\mathrm{M} / \mathrm{L}) \odot$ (dashed line) or $\sigma_{8} \simeq 0.7$ (dot-dashed line) Lower panels: Luminosity of the brightest galaxy in each group as function of group mass. Results are shown for both the 2dFGRS (left) and MGRS (right) group catalogues. Errorbars indicate 1- $\sigma$ scatter around the mean. Solid lines indicate two power-law relations, and are shown to facilitate a comparison.

\section{Galaxy Groups}

We recently also developed a halo-based group finder that can successfully assign galaxies into groups according to their common haloes [18]. The basic idea behind our group finder is similar to that of the host-satellite selection criteria discussed above. In short, we start with an assumed mass-to-light ratio to assign a tentative mass to each potential group. This mass is used to estimate the size and velocity dispersion of the underlying halo that hosts the group, which in turn is used to determine group membership (in redshift space). This procedure is iterated until group 
memberships converge. Detailed tests with our MGRS show that this group finder (i) is $\gtrsim 90 \%$ complete in terms of group membership, (ii) yields interloper fractions $\lesssim 20 \%$, and (iii) yields group catalogues that are insensitive to the initial assumption of the mass-to-light ratios.

Applying this group finder to the 2dFGRS yields a catalogue of 78708 groups, of which 7251 are binaries, 2343 are triplets, and 2502 contain four members or more. Group masses, $M$, are determined by computing the mean separation between all groups brighter than the group under consideration and matching this with the mean separation between dark matter haloes more massive than $M$ (see [20] for details).

The upper left-hand panel of Fig. 3 plots the average number of group members found as function of group mass. The dashed line indicates the true $\langle N\rangle(M)$ computed directly from the CLF used to construct the MGRS. The solid line shows the occupation numbers obtained from the groups selected from the MGRS using our halo-based group finder. Note the excellent agreement with the 'input' values, indicating that our group finder is very reliable. The dotted line indicates the group occupation statistics obtained from our 2dFGRS group catalogue. Clearly, the CLF predicts too many galaxies per group of a given mass. This is also evident from the upper right-hand panel, where the number of groups is plotted as function of the number of group members. The solid histogram and solid line indicate the results for the 2dFGRS and our MGRS, respectively. Clearly, the MGRS, which is based on our CLF, predicts too many high-multiplicity groups. To remedy this, we need to either set the average mass-to-light ratio on the scales of clusters to $\sim 900 h(\mathrm{M} / \mathrm{L}) \odot$ (compared to $500 h(\mathrm{M} / \mathrm{L}) \odot$ for our fiducial model), or $\sigma_{8}$ has to be reduced to $\sim 0.7$. This is in excellent agreement with some of our previous results based on the pairwise peculiar velocity dispersions [19] and the abundances of satellite galaxies [15].

The lower panels of Fig. 3 plot the relation between the luminosity of the brightest (central) galaxy in each group, $L_{c}$, and the group (halo) mass, $M$. Results are shown both for groups in the 2dFGRS (left panel) and for those in our fiducial MGRS (right panel). The mean $L_{c}-M$ relation is remarkably similar for both samples, and well described by a broken power-law with $L_{c} \propto M^{2 / 3}$ at $M \lesssim 10^{13} h^{-1} M_{\odot}$ and $L_{c} \propto M^{1 / 4}$ at $M \gtrsim 10^{13} h^{-1} M_{\odot}$. At the low-mass end, this is in excellent agreement with results based on galaxy-galaxy weak lensing (e.g., [17]). At the massive end, $L_{c}$ only increases very slowly with halo mass, indicating that there must be a physical process that prevents the central galaxies in massive haloes from growing.

Finally, we want to emphasize that the groups can also be used to directly measure the halo (= group) occupation statistics. In [22] we present some of these results, and show that the CLF of 2dFGRS galaxies is perfectly consistent with a Schechter form, contrary to a recent claim by [23].

\section{Conclusions}

We have introduced a new statistic, the conditional luminosity function $\Phi(L \mid M)$, which is ideally suited to describe the galaxy dark matter connection. The CLF is well constrained using data on the clustering properties of galaxies as function of luminosity, and yields a direct link between light and mass, which is in excellent agreement with the kinematics of satellite galaxies.

Using the CLF, we have identified two characteristic scales in galaxy formation (cf. [4]). One at $M \sim 10^{11} h^{-1} \mathrm{M}_{\odot}$, where galaxy formation is most efficient, and one at $M \sim 10^{13} h^{-1} \mathrm{M}_{\odot}$, where 
a transition occurs from systems dominated by one brightest, central galaxy to systems with several dominant galaxies of comparable luminosity.

Using the CLF we have also constructed detailed mock galaxy redshift surveys which can be compared on a one-to-one basis to the 2dFGRS. The main disagreements between the MGRS and the 2dFGRS concern the pairwise preculiar velocity dispersions (see [19]), the abundances of satellite galaxies (see [15]), and the group multiplicity function (see Fig. 3). These are all reflections of one and the same problem: the $\Lambda \mathrm{CDM}$ concordance cosmology predicts too many, large groups and clusters, unless the average mass-to-light ratio of clusters in the photometric $b_{J^{-}}$ band is $\langle M / L\rangle_{\mathrm{cl}} \sim 900 h(\mathrm{M} / \mathrm{L})_{\odot}$ (with $\left.\sigma_{8}=0.9\right)$ or $\sigma_{8} \simeq 0.75$ (with $\left.\langle M / L\rangle_{\mathrm{cl}} \sim 500 h(\mathrm{M} / \mathrm{L})_{\odot}\right)$. Recently, these findings have been confirmed by a similar but independent study of the galaxy clustering properties in the SDSS [10].

\section{References}

[1] Benson A.J., Lacey C.G., Baugh C.M., Cole S., Frenk C.S., 2002, MNRAS, 333, 156

[2] Berlind A.A., Weinberg D.H., 2002, ApJ, 575587

[3] Brainerd T.G., 2004, preprint (astro-ph/0411244)

[4] Dekel A., 2004, preprint (astro-ph/0401503)

[5] Madgwick D.S., et al., 2002, MNRAS, 333, 133

[6] Mo H.J., White S.D.M., 1996, MNRAS, 282, 347

[7] Mo H.J., Yang X., van den Bosch, F.C., Jing Y.P., 2004, MNRAS, 349, 205

[8] Norberg P., et al., 2002, MNRAS, 332, 827

[9] Seljak U., 2000, MNRAS, 318, 203

[10] Tinker J., Weinberg D.H., Zheng Z., Zehavi I., 2004, preprint (astro-ph/0411777)

[11] Vale A., Ostriker J.P., 2004, MNRAS, 353, 189

[12] van den Bosch F.C., Yang X.H., Mo H.J., 2003, MNRAS, 340, 771

[13] van den Bosch F.C., Mo H.J., Yang X.H., 2003, MNRAS, 345, 923

[14] van den Bosch F.C., Norberg P., Mo H.J., Yang X.H., 2004, MNRAS, 352, 1302

[15] van den Bosch F.C., Yang X.H., Mo H.J., Norberg P., 2004, MNRAS, in press (astro-ph/0406246)

[16] Wang Y., Yang X.H., Mo H.J., van den Bosch F.C., Chu Y., 2004, MNRAS, 353, 287

[17] Yang X.H., Mo H.J., Kauffmann Guinevere, Chu Y.Q., 2003, MNRAS, 339, 387

[18] Yang X.H., Mo H.J., van den Bosch F.C., 2003, MNRAS , 339, 1057

[19] Yang X.H., Mo H.J., Jing Y.P., van den Bosch F.C., Chu Y.Q., 2004, MNRAS , 350, 1153

[20] Yang X.H., Mo H.J., van den Bosch F.C., Jing Y.P., 2004, MNRAS , in press (astro-ph/0405234)

[21] Yang X.H., Mo H.J., van den Bosch F.C., Jing Y.P., 2004, MNRAS , in press (astro-ph/0406593)

[22] Yang X.H., Mo H.J., Jing Y.P., van den Bosch F.C., 2004, MNRAS , preprint (astro-ph/0410114)

[23] Zheng Z., et al., 2004, ApJ, preprint (astro-ph/0408564) 